Théâtres d'Afrique : des traces aux archives

\title{
Théâtres d'Afrique : des traces aux archives
}

\section{Aurore Desgranges}

\section{OpenEdition}

\section{Journals}

Édition électronique

URL : https://journals.openedition.org/coma/4560

DOI : $10.4000 /$ coma.4560

ISSN : 2275-1742

\section{Éditeur}

Institut des textes \& manuscrits modernes (ITEM)

\section{Référence électronique}

Aurore Desgranges, "Théâtres d'Afrique : des traces aux archives », Continents manuscrits [En ligne]

13 | 2019, mis en ligne le 22 novembre 2019, consulté le 12 janvier 2023. URL : http:// journals.openedition.org/coma/4560 ; DOI : https://doi.org/10.4000/coma.4560

Ce document a été généré automatiquement le 12 janvier 2023.

\section{cc) (i) $(9)$}

Creative Commons - Attribution - Pas d'Utilisation Commerciale - Pas de Modification 4.0 International - CC BY-NC-ND 4.0

https://creativecommons.org/licenses/by-nc-nd/4.0/ 


\title{
Théâtres d'Afrique : des traces aux archives
}

\author{
Aurore Desgranges
}

1 Le motif du chercheur-enquêteur, investi dans une recherche active des traces de la création, s'invite dans les études théâtrales de manière particulièrement signifiante depuis les années 2000. L'ère de la « célébration de la trace ${ }^{1}$ » trouve son origine dans la critique génétique, approche résolument indiciaire ${ }^{2}$, apparue dans les années 1960 et qui très vite se pense comme une «science des processus ${ }^{3}$ ». Ces études, centrées sur l'amont de la représentation permettent de définir l'acte théâtral dans un mouvement d'interaction constant entre le texte et la scène ${ }^{4}$. L'auctorialité est envisagée comme un phénomène complexe, incarnée par le duo entre l'auteur et le metteur en scène, mais relève aussi d'une collectivité plus large qui intègre le pôle technique de la création ${ }^{5}$.

2 Si cet intérêt pour les processus de création encourage les mesures politiques de conservation et de numérisation des archives théâtrales, il renouvelle également les pratiques concrètes de recherche. En réunissant des matériaux hétéroclites (documents de travail artistique et administratif, observations de répétitions, captations, entretiens avec les artistes etc.), les chercheurs "fabriquent" des archives dans l'intention d'élaborer un projet spécifique ${ }^{6}$. Ce qui peut être qualifié de «tournant épistémologique ${ }^{7}$ » rappelant la porosité de la frontière entre la discipline des études théâtrales et celle de l'histoire mais aussi de l'anthropologie ${ }^{8}$, participe toutefois d'une certaine dissolution du sens juridique premier du terme « archives » tel que défini dans le code du patrimoine. L'exercice de l'activité d'une personne, d'un service, d'un organisme serait la condition de la cohérence de l'ensemble documentaire constitué par les archives 9 . Or, le sens du terme « archives » se trouve étendu ici, indéniablement associé à un ensemble de concepts attenants, aux contours plus flous, tels que celui de «trace » de la création dans des événements ou œuvres postérieurs à l'époque étudiée ou encore d'«archive vivante ${ }^{10}$ " en la personne des comédiens dépositaires de traditions de jeu et de pratiques.

3 Cette extension d'usage fait écho à la place croissante prise par le paradigme de l'enquête dans la littérature contemporaine ${ }^{11}$ ou au développement d'un imaginaire de 
l'archive dans le sillage de Derrida et Farge ${ }^{12}$, donnant lieu à des emplois variés des archives dans différentes formes artistiques ${ }^{13}$. Elle engage du moins la recherche scientifique sur un chemin qui promet d'être aussi prolifique qu'il est problématique. Se pose très vite la question de la démarcation entre travail mémoriel et travail historique ${ }^{14}$ ou encore celle de l'influence de la présence du chercheur sur les archives constituées, de leur sélection à leur valorisation ${ }^{15}$...

Ce numéro de Continents Manuscrits souhaite ré-affirmer le potentiel heuristique de telles démarches en rappelant qu'elles ont été cruciales pour la visibilité des théâtres d'Afrique, permettant aussi bien de valoriser des formes « jugées mineures ${ }^{16}$ » que des auteurs ou des œuvres reconnus uniquement à une échelle nationale. C'est ainsi qu'Alain Ricard, Karin Barber et John Collins ont pu reconstituer l'histoire sociale des formes de théâtre populaire dans les années 1960-1970 au Ghana, Togo et Nigéria à partir d'observations participantes et d'un travail de répertorisation des textes et performances ${ }^{17}$. Jean-Pierre Guingané, jouissant alors d'un positionnement double de chercheur et d'artiste metteur en scène, comédien, auteur, réalise une immense collecte de textes dramatiques à partir des années 1970 au Burkina Faso dont ses deux thèses rendent compte ${ }^{18}$. Il documente la production dramatique sur le territoire dans un contexte de faible soutien étatique à la production culturelle.

5 L'exploration en cours du fonds d'archives de Bernard Zadi Zaourou par Dominique Traoré, Marie-Clémence Adom, Adama Coulibaly (etc.) de l'Université Félix HouphouëtBoigny, relève d'une démarche similaire. En cherchant à sensibiliser les autorités ivoiriennes à la conservation d'un patrimoine théâtral national, les porteurs du projet souhaitent combler un vide historiographique autour des artistes qui se sont surtout distingués à une échelle nationale, au croisement du champ politique et du champ culturel.

6 En outre, de telles démarches permettent de revenir sur des événements majeurs en révélant de nouvelles archives, porteuses de mémoires dissidentes ou contrastées. La recherche de Dominique Malaquais, Éloi Ficquet, Malika Rahal et Cédric Vincent sur les festivals panafricains des années 1960-1970 s'inscrit dans cette perspective ${ }^{19}$.

7 Les mêmes logiques d'invisibilisation perdurent pour la documentation de la création contemporaine dans la mesure où la plus grande partie des études critiques se concentre sur les artistes reconnus comme auteurs dramatiques et réfléchissent les enjeux esthétiques à partir des pièces éditées principalement en Europe, en raison de difficultés de circulation et de diffusion de l'édition africaine ${ }^{20}$. Ce constat constitue le point de départ des travaux de Maëline Le Lay qui s'attachent à resituer dans leur contexte historique et géographique les pratiques de théâtre de sensibilisation à partir de répertoires plurilingues de textes établis in situ ${ }^{21}$.

Dans le sillage de ces travaux, les contributions réunies dans ce numéro interrogent donc les modalités de constitution des archives théâtrales africaines.

9 Fernand Nouwligbeto, à travers l'étude de la pièce Zojagé (1974) créée par l'Ensemble artistique national du Bénin, souligne l'intérêt à revenir sur des créations qui ont été considérées comme inféodées au projet idéologique révolutionnaire (1972-1989). L'analyse du canevas de création, mis en relation avec des entretiens avec les artistes et des documents de l'après-scène, révèle une intrication complexe des différents enjeux politiques et esthétiques. L'intérêt porté à la question des archives et des traces permet de renouveler l'étude esthétique de créations envisagées jusqu'alors exclusivement sous l'angle de l'anthropologie. C'est selon Brian Valente-Quinn la raison pour laquelle 
la création théâtrale ambulante Bamba Mos Xam, en tournée nationale dans les années 1960-1970, consacrée à l'œuvre de Cheick Amadou Bamba, saint fondateur soufi de la confrérie mouride au Sénégal, n'a pas fait l'objet d'étude scientifique. À partir de l'empreinte laissée par le spectacle dans la mémoire des spectateurs et acteurs culturels, et d'un travail de retranscription de la pièce, il rend compte des innovations portées par la mise en scène et le jeu d'acteur.

La question des modalités de transmission entre différentes générations artistiques est abordée dans l'article d'Aurore Desgranges autour de l'héritage laissé par le romancier et journaliste Norbert Zongo assassiné en 1998 au Burkina Faso. Sa production intellectuelle étroitement liée à son combat politique forment une " matière " faisant l'objet de nombreux prolongements et réappropriations dans la création contemporaine, comme le laisse voir l'étude de textes réunis dans le cadre d'une archive «fabriquée » sur le terrain ouagalais entre 2015 et 2018. Maëline Le Lay revient sur ses travaux de recherche menés au Katanga (République démocratique du Congo) et dans la région des Grands Lacs et réfléchit aux enjeux méthodologiques et théoriques de la pratique de recherche littéraire et théâtrale sur le terrain. En s'intéressant à l'influence de l'enquête sur la constitution de l'objet d'étude ainsi qu'au geste de transformation des textes étudiés sur le terrain, elle renouvelle et enrichit le questionnement sur « les récits d'archives ${ }^{22}$ ».

11 L'approche archivistique permet par ailleurs de considérer pour elles-mêmes, les instances de consécration qui font que le "théâtre africain " s'est formé en tant qu'objet d'étude. Céline Gahungu présente par le biais d'une chronique les commencements d'un travail de recherche sur les archives du concours théâtral interafricain créé en 1967 par l'Office de coopération radiophonique (OCORA). Elle pose ainsi les premiers jalons d'une histoire littéraire, théâtrale et sociale. Enfin, l'entretien avec Nicolas Martin-Granel et Julie Peghini nous fait entrer dans la fabrique des archives théâtrales de l'auteur et metteur en scène Sony Labou Tansi. Ils reviennent sur la première étape de leur travail exposée dans l'ouvrage La Chair et l'Idée ${ }^{23}$ et exposent les enjeux de reconstitution d'un fonds d'archive dispersé et fondamentalement hétéroclite.

\section{BIBLIOGRAPHIE}

BARBER Karin, COLLINS John, RICARD Alain, West african popular theatre, Bloomington and

Indianapolis, Indiana University Press, 1997.

DE BIASI Pierre-Marc, Génétique des textes, CNRS Éditions, coll. « Biblis », Paris, 2011.

DEMANZE Laurent, Un nouvel âge de l'enquête, Éditions Corti, coll. « Les Essais », Paris, 2019.

DENIZOT Marion, « L'engouement pour les archives du spectacle vivant », Écrire l'histoire [en ligne], $\mathrm{n}^{\circ}$ 13-14, 2014, http://journals.openedition.org/elh/475, consulté le 15 octobre 2019.

GINZBURG Carlo, «Signes, traces, pistes : Racines d'un paradigme de l'indice », Le Débat 1980/6, $\mathrm{n}^{\circ}$ 6, p. 3-44.

GRÉSILLON Almuth, MERVANT-ROUX Marie-Madeleine, BUDOR Dominique, Genèses théâtrales, CNRS 
Editions, coll. « Art/cinéma », Paris, 2010.

FIQUET Éloi, MALAQUAIS Dominique, RAHAL Malika, VINCENT Cédric, « Panafest : une archive en devenir », LE LAY Maëline, MALAQUAIS Dominique, SIEGERT Nadine, dir., Archives (remix), Vues d'Afrique, Presses universitaires de Rennes, Rennes, 2015, p. 209-228.

GRÉSILLON Almuth, MERVANT-ROUX Marie-Madeleine, BUDOR Dominique, Genèses théâtrales, CNRS Éditions, Paris, 2010.

LE LAY Maëline, "La parole construit le pays », Théâtre langue et didactisme au Katanga, (République démocratique du Congo), Honoré Champion, coll. « Francophonies », Paris, 2014.

LE LAY Maëline, MALAQUAIS Dominique, SIEGERT Nadine, Archives (re)mix, Vues d'Afrique, Presses Universitaires de Rennes, Rennes, 2015.

LUCET Sophie, «Les instruments de la refiguration du temps, Traces, héritages et mémoires du spectacle vivant ", Mémoires, traces et archives en création dans les arts de la scène, LUCET Sophie, PROUST Sophie dir., Presses universitaires de Rennes, Rennes, 2017, p. 13-26.

LUCET Sophie, PROUST Sophie dir., Mémoires, traces et archives en création dans les arts de la scène, Presses universitaires de Rennes, Rennes, 2017.

MAC AULEY Gay, « Towards an ethnography of rehearsal », New Theatre Quaterly, vol. 14, Issue 53, p. 75-85, 1998.

MARTIN-GRANEL Nicolas, PEGHINI Julie, dir., Sony Labou Tansi en scène (s), La Chair et l'Idée, Théâtre et poèmes inédits, lettres, témoignages, écrits et regards critiques, Les solitaires intempestifs, Besançon, 2015.

MERVANT-ROUX Madeleine, « Construire à plusieurs la machine à jouer », Genesis, n 41, Créer à plusieurs mains, 2015.

PIÉGAY-GRos Nathalie, « Récits d'archive », Écrire l'histoire [en ligne], n 13-14, 2014, https:// journals.openedition.org/elh/473, consulté le 10 novembre 2019.

SIAUD Florent, «La génétique de la mise en scène à l'épreuve de l'expérience humaine », ANOKHINA Olga, IDMHAND Fatiha dir., La Fabrique du texte à l'épreuve de la génétique, Éditions des archives contemporaines, France, 2018, p. 29-43.

THIERRY Raphaël, Le Marché du livre africain et ses dynamiques littéraires, le cas du Cameroun, Pessac, Presses universitaires de Bordeaux, coll. « Littérature des Afriques », 2015.

ZENETTI Marie-Jeanne, « Détournements d'archives : littérature documentaire et dialogue interdisciplinaire ", article issu d'une communication au colloque « Les écritures des archives, I : Littérature, discipline littéraire et archives », organisé par LouIs Annick et ZGoLA Clara, 13-14 janvier 2017, EHESS-Université Paris Sorbonne, à paraître sur le site fabula.fr.

\section{NOTES}

1. Sophie Lucet, «Les instruments de la refiguration du temps. Traces, héritages et mémoires du spectacle vivant ", Mémoires, traces et archives en création dans les arts de la scène, Sophie Lucet, Sophie Proust, dir., Presses universitaires de Rennes, Rennes, 2017, p. 13-26.

2. Carlo Ginzburg, «Signes, traces, pistes: Racines d'un paradigme de l'indice », Le Débat, $\mathrm{n}^{\circ} 6$, 1980/6, p. 3-44.

3. Pierre-Marc De Biasi, Génétique des textes, CNRS Éditions, coll. « Biblis », Paris, 2011.

4. Almuth Grésillon, Marie-Madeleine Mervant-Roux, Dominique Budor, Genèses théâtrales, CNRS Éditions, coll. « Art/cinéma », Paris, 2010.

5. Madeleine Mervant-Roux, «Construire à plusieurs la machine à jouer ", Genesis, n 41, « Créer à plusieurs mains », 2015, https://journals.openedition.org/genesis/1525, consulté le 10 novembre 2019. 
6. Marion Denizot, «L'engouement pour les archives du spectacle vivant », Écrire l'histoire [en ligne], n 13-14, 2014, http://journals.openedition.org/elh/475, consulté le 10 novembre 2019.

7. Ibidem.

8. Gay Mac Auley, "Towards an ethnography of rehearsal», New Theatre Quaterly, vol.14, Issue 53, p.75-85, 1998 ; Florent Siaud, «La génétique de la mise en scène à l'épreuve de l'expérience humaine », Olga Anokhina, Fatiha Idmhand, dir., La Fabrique du texte à l'épreuve de la génétique, Éditions des archives contemporaines, France, 2018, p. 29-43.

9. Je me réfère à la distinction établie par Marion Denizot entre archives héritées et archives fabriquées à partir de la définition du terme «archives » telle qu'elle apparaît dans le code du patrimoine (art. L. 211-1), ibid.

10. Sophie Lucet, Sophie Proust, dir., Mémoires, traces et archives en création dans les arts de la scène, Presses universitaires de Rennes, Rennes, 2017

11. Laurent Demanze, Un nouvel âge de l'enquête, Éditions Corti, coll. « Les Essais », Paris, 2019.

12. Marie-Jeanne Zenetti, «Détournements d'archives: littérature documentaire et dialogue interdisciplinaire", article issu d'une communication au colloque «Les écritures des archives, I : Littérature, discipline littéraire et archives», organisé par Annick Louis et Clara Zgola, 13-14 janvier 2017, EHESS-Université Paris Sorbonne, à paraître sur le site fabula.fr.

13. Maëline Le Lay, Dominique Malaquais, Nadine Siegert, dir., Archives (remix), Vues d'Afrique, Presses universitaires de Rennes, Rennes, 2015, p. 209-228.

14. Nathalie Piégay-Gros, «Récits d'archive ", Écrire l'histoire [en ligne], nº 13-14, 2014, https:// journals.openedition.org/elh/473, consulté le 10 novembre 2019.

15. Marion Denizot, op. cit.

16. Ibidem.

17. Karin Barber, Johns Collins, Alain Ricard, West African Popular Theater, Bloomington and Indianapolis, Indiana University Press, 1997.

18. Jean-Pierre Guingané, Le Théâtre au Haute-Volta, structure-production-diffusion-public, Thèse pour le doctorat de troisième cycle, Bordeaux III, 1978; Jean-Pierre Guingané, Théâtre et développement culturel en Afrique: le cas du Burkina Faso, Thèse pour le doctorat ès sciences de l'information et de la communication, Bordeaux III, 1987.

19. Éloi Fiquet, Malaquais Dominique, Malika Rahal, Cédric Vincent, «Panafest : une archive en devenir ", Maëline Le Lay, Dominique Malaquais, Nadine Siegert, dir., Archives (remix), Vues d'Afrique, Presses universitaires de Rennes, Rennes, 2015, p. 209-228.

20. Raphaël Thierry, Le Marché du livre africain et ses dynamiques littéraires, le cas du Cameroun, Pessac, Presses universitaires de Bordeaux, coll. « Littérature des Afriques », 2015.

21. Maëline Le Lay, "La parole construit le pays», Théâtre langue et didactisme au Katanga (République démocratique du Congo), Honoré Champion, coll. « Francophonies », Paris, 2014.

22. Nathalie Piégay-Gros, op. cit.

23. Nicolas Martin-Granel, Julie Peghini, dir., Sony Labou Tansi en scène (s), La Chair et l'Idée, Théâtre et poèmes inédits, lettres, témoignages, écrits et regards critiques, Les solitaires intempestifs, Besançon, 2015. 
INDEX

Mots-clés : traces, archives, visibilité, théâtres d'Afrique

Keywords : traces, archives, visibility, theater in Africa

\section{AUTEUR}

\section{AURORE DESGRANGES}

Doctorante à l'université Lyon II (Passages XX-XXI) et à l'Université Ouaga I Joseph Ki-Zerbo (LES, Littératures Espaces Sociétés), chercheuse associée à l'équipe « Manuscrits francophones » de l'ITEM (CNRS-ENS), AURORE DESGRANGES réalise une thèse sur les « Formes et enjeux du texte dramatique au Burkina Faso » sous la direction de Véronique Corinus et Salaka Sanou. 